\title{
Tuning of frictional properties in torsional contact by means of disk grading
}

\author{
Gianluca COSTAGLIOLA ${ }^{1}$, Federico BOSIA ${ }^{2}$, Nicola M PUGNO ${ }^{3,4, *}$ \\ ${ }^{1}$ Civil Engineering Institute, École Polytechnique Fédérale de Lausanne, Lausanne 1015, Switzerland \\ ${ }^{2}$ Department of Applied Science and Technology, Politecnico di Torino, Corso Duca degli Abruzzi 24, Torino 10129, Italy \\ ${ }^{3}$ Laboratory for Bioinspired, Bionic, Nano, Meta Materials \& Mechanics, Department of Civil, Environmental and Mechanical Engineering, \\ University of Trento, Trento 38123, Italy \\ ${ }^{4}$ School of Engineering and Materials Science, Queen Mary University of London, London E1 4NS, UK \\ Received: 08 November 2020 / Revised: 22 March 2021 / Accepted: 05 June2021 \\ (C) The author(s) 2021.
}

\begin{abstract}
The contact of two surfaces in relative rotating motion occurs in many practical applications, from mechanical devices to human joints, displaying an intriguing interplay of effects at the onset of sliding due to the axisymmetric stress distribution. Theoretical and numerical models have been developed for some typical configurations, but work remains to be done to understand how to modify the emergent friction properties in this configuration. In this paper, we extend the two-dimensional (2D) spring-block model to investigate friction between surfaces in torsional contact. We investigate how the model describes the behavior of an elastic surface slowly rotating over a rigid substrate, comparing results with analytical calculations based on energy conservation. We show that an appropriate grading of the tribological properties of the surface can be used to avoid a non-uniform transition to sliding due to the axisymmetric configuration.
\end{abstract}

Keywords: friction; torsion; numerical simulations; grading

\section{Introduction}

The configuration of two surfaces subjected to frictional sliding under torsion is commonly encountered in many mechanical devices. The joints of car suspensions are composed by steel bearings rotating over a flat surface [1], in which friction is responsible for non-trivial fretting wear phenomena [2-5], and other examples are found in spherical and slewing roller bearings, crankshafts, and center plates of train bogies [6-8]. Joints involving torsional rotation can also be found in the human body, e.g., in spinal disks [9], knee $[10,11]$ and hip articulations $[12,13]$, and in bio-medical implants [14, 15]. For this reason, torsional contact has been investigated in experimental studies reproducing these systems [16, 17].

Moreover, this configuration is intrinsically interesting for experiments involving fundamental aspects of friction between soft and rigid surfaces [18-22]. The main reason lies in the non-uniform stress distribution induced by rotation, so that a differential surface slip takes place. While the outer circumference borders are undergoing slippage, inner surface regions may not be doing so. Thus, torsional contact has been used to verify early theoretical predictions of partial surface slips in the Hertzian theory for a sphere on a plane under a torque and a normal force [23-25].

More recent experimental works and theoretical models have focused on adhesion [26], viscoelasticy [27-29], and effects of surface patterning [30]. These studies have mainly considered a soft half plane with a rigid rotating punch, so that further theoretical and numerical work is required to address remaining questions regarding the transition to sliding or non-

* Corresponding author: Nicola M PUGNO, E-mail: nicola.pugno@unitn.it 
trivial geometrical features such as surface textures, whose contact leads to complex friction behaviour due to the axisymmetric configuration.

Recent studies have highlighted a complex interplay of effects at the onset of frictional sliding in nominally flat surfaces [31, 32]. These effects have been captured by simplified spring-block models, providing useful insights on basic microscopic phenomena determining emergent behavior [33-35]. A formulation of the spring-block model has been used to address the transition to sliding in the presence of non-uniform surface stress distributions induced by surface patterning [36-38]. The model has qualitatively explained the static friction reduction observed in recent experiments [39-45]. For these reasons, we expect to derive, using these models, useful information in the case of torsional contact, which could be useful for the manipulation of emergent frictional properties in this type of system. In particular, in Refs. [46, 47], we have investigated how an appropriate grading of surface tribological properties can be useful to manipulate macroscopic friction. We expect that these concepts can be applied to the present case.

In this paper, we extend the two-dimensional (2D) spring-block model developed to torsional contact, to investigate the radial dependence of observables, the onset of sliding and effects due to surface grading [38]. Our study aims to find useful insights that can be exploited in practical applications in which a modification of standard torsional friction behavior of torsional contact is required.

The paper is divided as follows: in Section 2, we present the model and the setup for studying torsional contact; in Section 3, we illustrate the transition to sliding that emerges from the model, comparing it with analytical calculations and literature results; in Section 4 , we show the effect of the surface grading on the emergent friction properties, in Section 5 we provide the conclusions.

\section{Model}

We consider a flat elastic two dimensional circular surface slowly rotating at constant angular velocity around its vertical axis over a rigid substrate, approximating a cylinder whose thickness is much smaller than the radius (Fig. 1). The surface is discretized into a mesh of springs and masses, as shown in the enlargement of Fig. 1, also used in Ref. [38]. We will indicate this as spring-block model (SB).

To implement an axisymmetric setup, we adopt the following procedure. We start from a square mesh of $N \times N$ blocks, whose internal distance along both axes is $l$. The block position inside the mesh can be labeled using two integer indexes $a=(n, m)$, with $-N / 2 \leq n, m \leq N / 2-1$ along the $x$ and $y$ axes, respectively. At the beginning, the mesh center of mass is placed at the origin of the coordinate system,

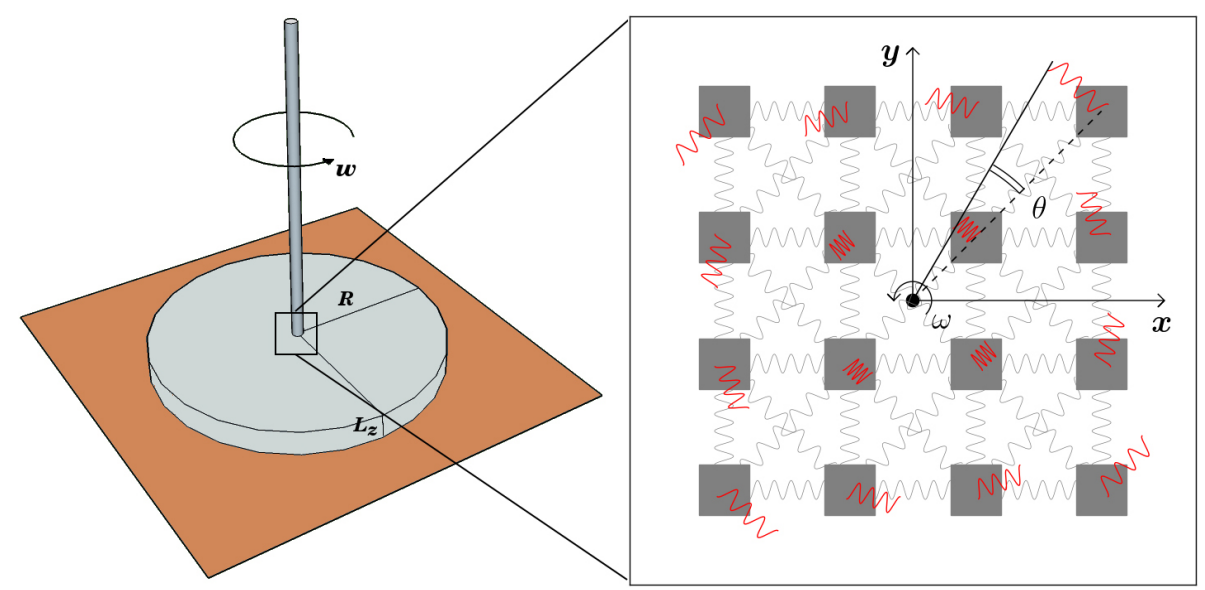

Fig. 1 Schematic abstraction of the considered configuration. A thin elastic cylindrical surface (represented by the grey disk), rotating around the vertical axis passing from its center and in torsional contact over a rigid substrate (represented by the orange plane). A uniform pressure is applied over the whole disk surface. The spring mesh used to model the contact problem is shown in the enlargement, with internal springs between blocks (grey) and shear springs attached to the rotor (red). Coordinate axes, rotation angle $\theta$ and angular velocity $\omega$ are also indicated. 
so that the position vector of the block $i=(n, m)$ is $\boldsymbol{r}_{i}=(l(0.5+n), l(0.5+m))$. In this way, the rotational vertical axis passing through the origin will not intersect any block. To reproduce a circular surface using a square mesh, we eliminate all blocks whose distance from the origin is greater than the radius $R \equiv l \cdot N / 2$. Thus, for sufficiently large $N$ we obtain a disk approximated by small square elements.

The equivalence of the spring mesh with a homogeneous isotropic elastic material of Young's modulus $E$ and Poisson's ratio $v=1 / 3$ can be imposed by following the procedure illustrated in Ref. [48] applied to our case. The stiffness of springs connecting the nearest neighbors blocks is $K_{\text {int }}=3 / 4 E L_{z}$, where $L_{z}$ is the thickness of the layer, and the stiffness of springs connecting to the next-nearest neighbors must be $K_{\text {int }} / 2$ [35]. Hence, the internal elastic force on a generic block $i$ exerted by the neighbor $j$ is

$$
\boldsymbol{F}_{\text {int }}^{(i, j)}=k_{i j}\left(r_{i j}-l_{i j}\right)\left(\boldsymbol{r}_{j}-\boldsymbol{r}_{i}\right) / r_{i j}
$$

where $r_{i j}$ is the modulus of the distance between $i$ and $j, l_{i j}$ is the modulus of the rest distance, and $k_{i j}$ is the stiffness of the spring connecting them.

All blocks are attached, through shear springs of stiffness $K_{\mathrm{s}}$, to a massless rotor subjected to uniform rotational motion around the vertical axis. In this formulation, the rotor plays the same role of the slider in the linear motion of the model described in Ref. [38]. The position vector of the rotor point attached to the block $i$ at time $t$ can be written as $\boldsymbol{r}_{i}^{s} \equiv$ $\left(\left(r_{i}^{0}\right)_{x} \cos (\omega t)-\left(r_{i}^{0}\right)_{y} \sin (\omega t),\left(r_{i}^{0}\right)_{x} \sin (\omega t)+\left(r_{i}^{0}\right)_{y} \cos (a t)\right)$, where $\left(r_{i}^{0}\right)_{x},\left(r_{i}^{0}\right)_{y}$ are the $x, y$ components of the position vector of the block $i$ at time zero and $\omega$ is the constant angular velocity. The rotation angle of the rotor is defined as $\theta \equiv \omega t$. Thus, the shear force on the $i$-th block given by the circular motion is

$$
\boldsymbol{F}_{\mathrm{s}}^{(i)}=K_{\mathrm{s}}\left(\boldsymbol{r}_{i}^{\mathrm{s}}-\boldsymbol{r}_{i}\right)
$$

We define the total longitudinal force on the block $i$ as

$$
\boldsymbol{F}_{\mathrm{mot}}^{(i)}=\sum_{j} \boldsymbol{F}_{\mathrm{int}}^{(i j)}+\boldsymbol{F}_{\mathrm{s}}^{(i)}
$$

This force lies in the $x, y$ plane and represents the sum of internal spring forces and the shear force due to the rotational motion.
A viscous damping force is added to eliminate artificial block oscillations, i.e., $\boldsymbol{F}_{\mathrm{d}}^{(i)}=-\gamma m \dot{\boldsymbol{r}}_{i}$, where $\gamma$ is the damping frequency. Friction itself could provide sufficient damping and prevent artificial oscillations, but in the present study we will also consider a grading in which the friction coefficient is close to zero. In this case, the block may surpass its equilibrium position during sliding and then return back. An additional viscous damping term prevents this type of phenomenon. A uniform pressure $P$ is applied on the system along the vertical axis, so that each block is subjected to a normal force $F_{n}^{(i)}=P l^{2}$. The total normal force is $F_{\mathrm{n}}=\sum_{i} F_{n}^{(i)}$.

The interactions between the blocks and the rigid substrate are introduced by means of the classical Amontons-Coulomb (AC) friction force with a statistical dispersion on the friction coefficients [38]. While the block $i$ is at rest, the friction force $F_{\mathrm{fr}}^{(i)}$ opposes the total driving force, i.e., $\boldsymbol{F}_{\mathrm{fr}}^{(i)}=-\boldsymbol{F}_{\mathrm{mot}}^{(i)}$, up to a threshold value $\boldsymbol{F}_{\mathrm{fr}}^{(i)}=\mu_{\mathrm{s} i} \boldsymbol{F}_{\mathrm{n}}^{(i)}$, where $\mu_{\mathrm{s} i}$ is the static friction coefficient and $\boldsymbol{F}_{\mathrm{n}}^{(i)}$ is the normal force on $i$. When this limit is exceeded, a constant dynamic friction force opposes the motion, i.e., $\boldsymbol{F}_{\mathrm{fr}}^{(i)}=-\mu_{\mathrm{d} i} \boldsymbol{F}_{\mathrm{n}}^{(i)} \hat{\boldsymbol{r}}_{i}$, where $\mu_{\mathrm{d} i}$ is the dynamic friction coefficient and $\hat{r}_{i}$ is the velocity direction of the block.

The friction coefficients are extracted from a Gaussian statistical distribution to introduce a noise in the system and mimic statistical fluctuations of the local roughness. Without a statistical dispersion, sliding would start simultaneously from all points on the edges, but after the first slip, all points on the external edge would be stuck again, due to static friction, until the force threshold is exceeded with the rotation. Thus, there would be a sort of rotational stick-slip motion. The same happens with the spring-block model formulation in linear sliding. This is a completely different phenomenology, which is not the main focus of the study.

Therefore, we introduce the following statistical distribution on both static and dynamic friction coefficients. In the following, we will drop the subscript $\mathrm{s}$, d every time the considerations apply to both the coefficients.

$$
p\left(\mu_{i}\right)=(\sqrt{2 \pi} \sigma)^{-1} \exp \left[-\left(\mu_{i}-(\mu)_{\mathrm{m}}\right)^{2} /\left(2 \sigma^{2}\right)\right]
$$

where $(\mu)_{\mathrm{m}}$ denotes the mean of the microscopic friction coefficients and $\sigma$ its standard deviation. 
Although the occurrence of negative values or values such that $\mu_{\mathrm{d}_{i}}>\mu_{\mathrm{s} i}$ is a rare event, we check this possibility and, if verified, we discard the friction coefficients and new ones are extracted, until they satisfy the required conditions. Thus, the complete equation of motion for the $i$-th block is

$$
m \ddot{r_{i}}=\sum_{j} \boldsymbol{F}_{\mathrm{int}}^{(i j)}+\boldsymbol{F}_{\mathrm{s}}^{(i)}+\boldsymbol{F}_{\mathrm{fr}}^{(i)}+\boldsymbol{F}_{\mathrm{d}}^{(i)}
$$

The overall system of differential equations is solved using a fourth-order Runge-Kutta algorithm. In order to calculate the average of any observable, the simulation must be iterated, extracting each time new random friction coefficients. In repeated tests, an integration time step of $h=10^{-8} \mathrm{~s}$ proves to be sufficient to reduce integration errors under the statistical uncertainty in the range adopted for the parameters of the system.

Realistic macroscopic elastic properties are chosen, e.g., a Young's modulus $E=1 \mathrm{MPa}$, which is typical for a soft polymer or rubber-like material, and a density $\rho=1.2 \mathrm{~g} / \mathrm{cm}^{3}$. The cylinder sizes are $R=1 \mathrm{~cm}$ and $L_{z}=1 \mathrm{~mm}$, the pressure is $P=10 \mathrm{kPa}$ and the angular velocity is in the range $0.01 \mathrm{rad} / \mathrm{s}<w<0.2 \mathrm{rad} / \mathrm{s}$, as adopted in experiments of Ref. [26]. The damping parameter is an arbitrary parameter that does not influence the final results if it is fixed in the underdamped regime $\left(\gamma<\sqrt{K_{\mathrm{int}} / m}\right)$ [35] and, in our case the fixed value is $\gamma=5 \mathrm{~m} \cdot \mathrm{s}^{-1}$. The number of blocks $N$ will be specified in each case. A study of its influence as arbitrary model parameter will be provided in Section 2.2. For the friction coefficients, the quantity of effective interest is the ratio between static and dynamic coefficient, so that we fix conventionally the static one to $\left(\mu_{\mathrm{s}}\right)_{\mathrm{m}}=1.0$ and perform the simulations varying the dynamic one in the range $0.5<\left(\mu_{\mathrm{d}}\right)_{\mathrm{m}}<0.8$, covering most of the measured values of the ratio for standard materials. Their standard deviations are set to five percent.

\subsection{Observables}

The quantity that is measured for the setup in Fig. 1 in an experimental apparatus can be identified as the total torque $\boldsymbol{M} \equiv \sum_{i} \boldsymbol{r}_{i} \times \boldsymbol{F}_{\mathrm{s}}^{(i)}$, i.e., the sum over all blocks of the vector product between the distance from the center and the force exerted by the rotor (Eq. (2)). Since both the force and the distance vectors lie in the horizontal plain, the torque has only one non-zero component that will be indicated with $M$.

The rotation angle of the block $i$ is indicated with $\theta_{i}$. In the absence of friction, this is equal to the nominal rotation angle $\theta$ of the rotor, but in the presence of friction we may expect $\theta_{i}<\theta$ for all $i$ values. We define the rotation lag $\Delta_{i} \equiv \theta-\theta_{i}$, i.e., the angular difference between the nominal rotation and the effective rotation undergone by $i$. Moreover, we define the critical angle $\theta_{\mathrm{c}}^{(i)}$ as the rotation angle corresponding to the first sliding of the block $i$, i.e., the first transition from static to dynamic friction, which can be deduced from the equation of motion.

Finally, we define the normalized surface force field $f$, i.e., the modulus of the total longitudinal driving force of Eq. (3) applied to the $i$-th block normalized by its normal force: $f^{i} \equiv\left|\boldsymbol{F}_{\text {mot }}^{(i)}\right| / F_{\mathrm{n}}^{(i)}$.

It is convenient to express quantities having an average axisymmetric distribution as a function of the distance from the center $r$, so that they can be averaged on all blocks placed at the same distance. For example, the torque $M(r)$ is calculated in a thin annulus of radius $r$, i.e., the sum on the blocks $i$ is restricted to those having a distance $r_{i}$ from the center such that $\left|r_{i}-r\right|<l$. Similarly, we define the quantities $\Delta(r)$, $\theta_{\mathrm{c}}(r)$, and $f(r)$, i.e., average rotation lag, critical angle, and applied force, respectively, of points located at distance $r$ from the center.

\subsection{Trend with $N$}

In Fig. 2, we show the typical behavior of the total torque $M$ as a function of time. It is useful to define the effective static friction coefficient $\left(\mu_{\mathrm{s}}\right)_{M}$ as the value reached by the first peak of the torque normalized with the disk radius and the applied normal force, and the dynamic one $\left(\mu_{\mathrm{d}}\right)_{M}$ as the average of the curve after therotation onset. These quantities characterize the behavior of $M$, but they must not be compared with the friction coefficients measured with experiments of linear sliding, since frictional force depends on the radial coordinate.

In the inset of Fig. 2, we show the influence of discretization, i.e., number of blocks $N$ determining the discretization length $l=R / N$, on the friction coefficients. Both these quantities are decreasing with $N$, but variations are limited to few percent. For other 


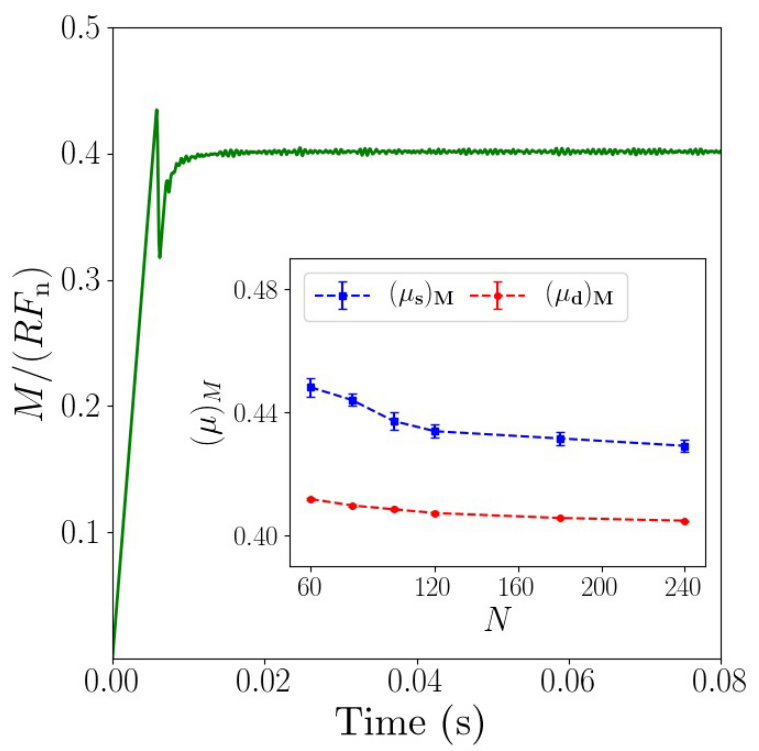

Fig. 2 Time evolution of the total normalized torque for $N=120$ and $\omega=0.2 \mathrm{rad} / \mathrm{s}$. In the inset, the effective friction coefficients as a function of $N$, showing variations within a few percent.

observables, effects of discretization are also negligible. Thus, we conclude that $N=120$ is sufficient to reduce discretization errors below statistical uncertainties for the main observables and that our general qualitative conclusions are not influenced by this issue.

\section{Benchmark results}

\subsection{Transition to sliding}

Due to the axisymmetric configuration, a non-uniform stress distribution arises before sliding. Applied forces are directed tangentially with respect to the rotation and are linearly increasing in modulus with the radius. Slippage starts from surface points on the outer circumference with smaller static friction coefficient values and involves the external part of the disk. However, this initial sliding does not involve the whole surface and stops at some point between the outer circumference and the center, where the tangential force is no longer large enough to trigger a further slip avalanche. After this phase, the external parts of the disk rotates at uniform velocity, while the central part slides gradually towards the center.

This sequence is illustrated in Fig. 3. Figure 3(a) shows the surface force field as a function of the radial distance from the center and the rotation angle $\theta$, while Fig. 3(b) shows the torque as a function of the rotation angle $\theta$. The peak of the total torque occurs when the maximum stress is reached at the outer border, while after a readjustment, the stationary dynamic rotational motion takes place. For points towards the center, instead, dynamic motion takes place with an incoherent stick-slip motion of single blocks without a sharp transition. This is because, in the internal region, the stored elastic energy is smaller due to the axisymmetric stress distribution, and the dynamics of the internal part is dominated by the statistical noise on the static friction thresholds. This is also illustrated in Fig. 3(c), showing the critical angle
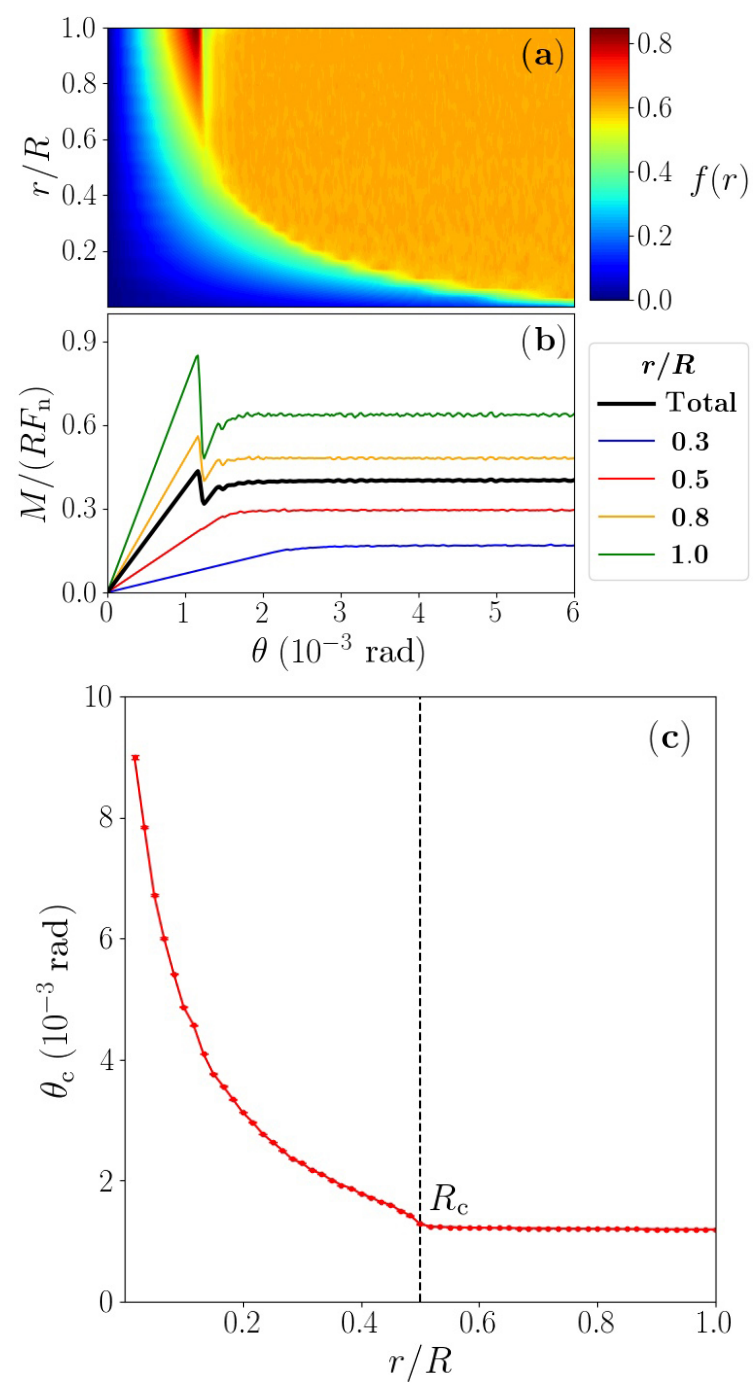

Fig. 3 (a) Normalized longitudinal force distribution $f(r)$ as a function of the radius (vertical axis) and the rotation angle (horizontal axis) $\theta=\omega t$; (b) evolution of the total torque (green line) and torque calculated in annular regions of radius $r$; (c) critical angle as a function of the radial coordinate. These simulations were performed with $N=120, \omega=0.2 \mathrm{rad} / \mathrm{s},\left(\mu_{\mathrm{d}}\right)_{\mathrm{m}} /\left(\mu_{s}\right)_{\mathrm{m}}=0.6$. 
$\theta_{c}(r)$ corresponding to each radial coordinate. The external part of the surface slips almost simultaneously at the same angle corresponding to the maximum stress peak.

The fractions of disk involved in the two phases are separated by a critical radius $R_{\mathrm{c}}$ : Points located at a greater distance from the center slips in the first phase and those located at a smaller distance in the second one. The critical radius can be estimated from the plot in Fig. 3(c), showing the curve of the critical angle corresponding to the radial coordinate: the critical radius corresponds to the "knee" of the curve, i.e., the radius where the slope abruptly increases. We have conventionally estimated this point as the radius in which the derivative of the curve exceeds a $5 \%$ percent of relative increase, as indicated in the plot.

\subsection{Comparison with analytical results and the literature}

In order to validate the model, we have developed analytical calculations based on energy conservation in a quasi-static regime. As shown in Section 3.1, the external part of the disk slides abruptly in the first phase of the transition to sliding, whereas the internal part slides gradually in a second phase. These regions are separated by a critical radius $R_{\mathrm{c}}$. Before any slip, elastic energy is accumulated due to the rotor. After the sliding of the external part, the elastic energy has been partially dissipated by friction, is partially still stored in the slider due to the angular lag $\Delta(r)$ with respect to the rotor, and is partially stored as shear elastic deformation of the disk itself. All these energies can be written in terms of the system elastic and frictional parameters, the critical radius $R_{\mathrm{c}}$, and the angular lag $\Delta(r)$. By imposing energy conservation and assuming that, after the first phase of the sliding, the system readjusts at the minimum energy, the quantities $R_{\mathrm{c}}$ and $\Delta(r)$ can be calculated. The detailed description of the calculation is reported in the Appendix.

In particular, from the calculation we can deduce that, in the limit of $L_{z}<R$, the critical radius is proportional to the ratio between dynamic and static friction, i.e., $R_{\mathrm{c}} / R=\mu_{\mathrm{d}} / \mu_{\mathrm{s}}$. The thickness introduces a correction, which can be also computed semi-analytically with the energy balance approach. As shown in Fig. 4(a), numerical results of the SB model confirm the linear trend and are close to the semi-analytical curve. Moreover, data approaches the semi-analytical curve for smaller velocities, as expected since calculations are performed in a quasi-static regime neglecting the rotational kinetic energy. For larger angular velocities, the critical radius decreases since the increase of the kinetic energy implies the simultaneous sliding of a larger surface fraction, i.e., smaller $R_{\mathrm{c}}$ value. Examples of critical angles as a function of the radius to estimate $R_{\mathrm{c}}$ are reported in Fig. 4(b) for various $\left(\mu_{\mathrm{d}}\right)_{\mathrm{m}}$.

Similar results can be found in the literature for axisymmetric systems, e.g., two elastic spheres in
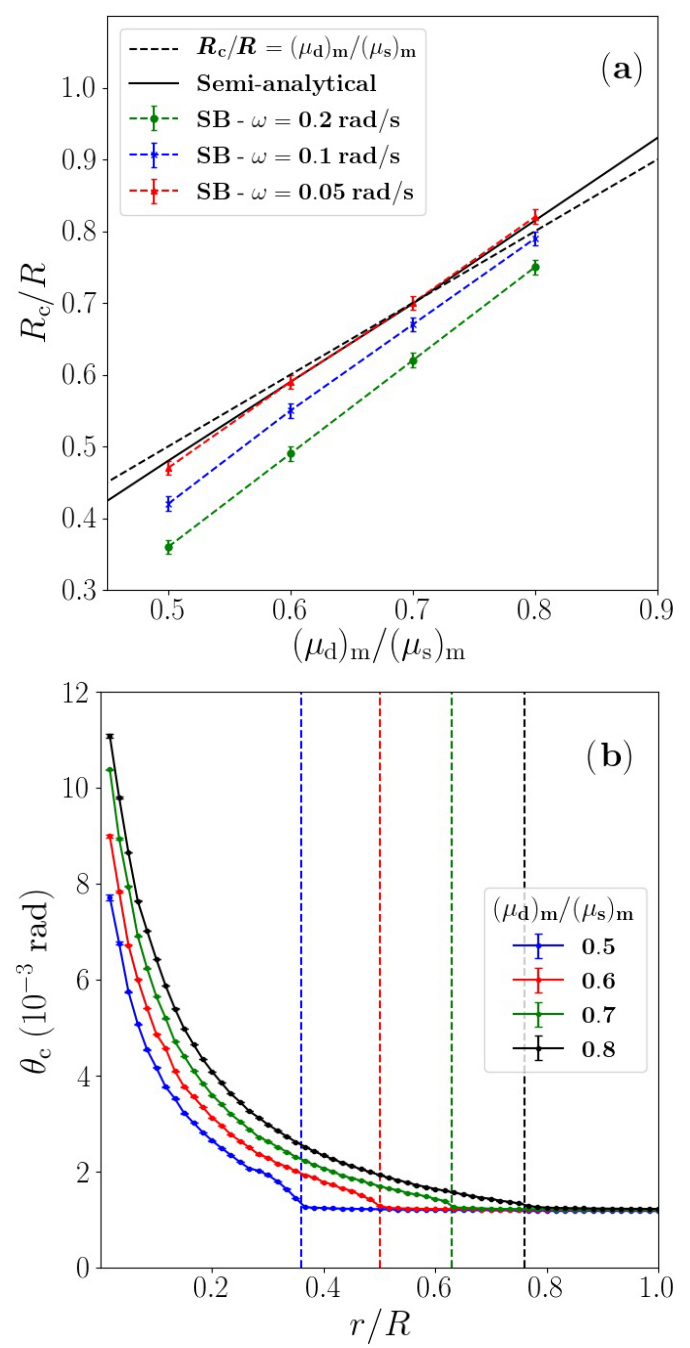

Fig. 4 (a) Critical radius as a function of the ration between friction coefficients for various angular velocities obtained with the SB model. These are compared with the predictions of analytical calculations reported in the Appendix. (b) Critical angle as a function of the radial coordinate for various ratios of the friction coefficients, allowing to estimate the critical radius of each curve (indicated by the dotted line). 
reciprocal rotation in the work by Lubkin [24]. According to his results, there is a critical radius separating the external sliding region and the internal region still at rest. In particular, this distance is a linear function of the dynamic friction coefficient, consistently with Fig. 4(a), confirming the general validity of our analytical and numerical results.

In Fig. 5, we report the macroscopic torque behavior for various ratios of the friction coefficients. The transition to sliding is qualitatively similar to that illustrated in Fig. 3, but the torque curve changes shape as the peak due to the sharp sliding of the external part disappears for larger dynamic friction. This is due to the reduced fraction of disk involved in this phase. Moreover, the curve for the torque with the dynamic friction coefficient closer to the static one is similar to that reported in Ref. [24], which, indeed, is obtained for a quasi-static rotation without static friction. In the stationary rotation regime, all curves for various dynamic friction coefficients collapse with a good approximation to the same value by dividing the normalized torque by $\left(\mu_{\mathrm{d}}\right)_{\mathrm{m}}$, as shown in the inset of Fig. 5. In Ref. [24], the value predicted for this quantity is $M /\left(\mu_{\mathrm{d}} R F_{\mathrm{n}}\right)=3 \pi / 16 \approx 0.59$, consistently with our estimates, ranging from 0.6 and 0.67 , depending on the sliding velocity.

Finally, The surface stress fields as a function of radius for various rotation angles are reported in Fig. 6, showing that the behavior is qualitatively similar to

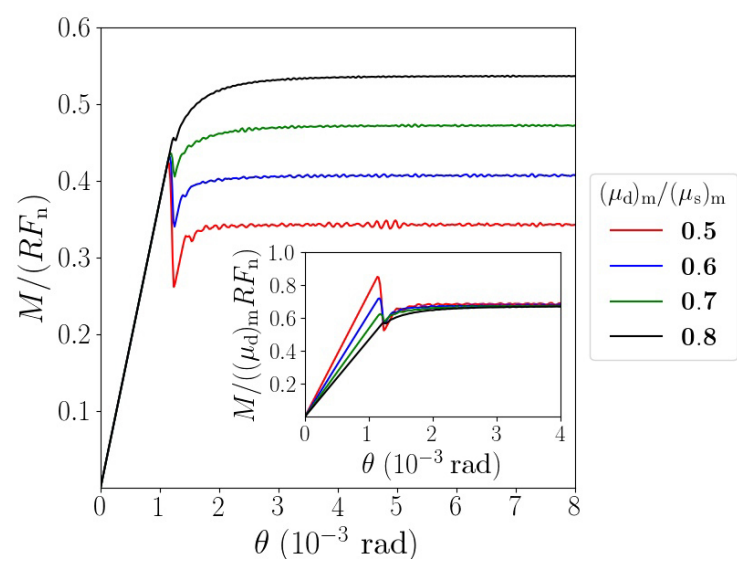

Fig. 5 Total torque for various ratios of microscopic friction coefficients. If the ratio between dynamic and static friction coefficients tends to one, the curve shape approaches the curve predicted in Ref. [24]. In the inset, the torque value after the transition to sliding can be rescaled to a constant value by dividing all curves by $\left(\mu_{\mathrm{d}}\right)_{\mathrm{m}}$. those observed in experimental work with a sphere rotating over a flat surface [26]. From all these results, we can conclude that the SB model can capture the underlying dynamics of similar axisymmetric systems observed in previous works.

\subsection{Angular lag}

In Section 3, we have analyzed the transition to sliding of the rotating surface, which can be divided in two parts: an abrupt slip of the external part of the disk, followed by a gradual transition to sliding towards the center, separated by acritical radius $R_{\mathrm{c}}$. Thus, the onset of sliding takes place at different times depending on the point location. This behavior induces a distortion of the elastic disk during the subsequent rotation: although all points rotate at the same angular velocity $\omega$, the angular lag $\Delta(r)$ is not a constant along the radius $r$. In other words, points towards the center slide at larger angular distance from the nominal rotation angle than those located at the border.

This is illustrated by the angular lag $\Delta(r)$, i.e., the angular distance between the nominal rotated angle $\theta$ by the rotor and the average rotation performed by the points at radius $r$. In Fig. 7(a), we report $\Delta(r)$ for the same disk at various times (rotation angle). The lag of the internal part of the disk, being still at rest, corresponds to $\theta$, and the fraction of disk at rest reduces as the rotation proceeds. For the external part, the lag decreases but is never zero due to friction. From analytical calculations reported in the Appendix, the

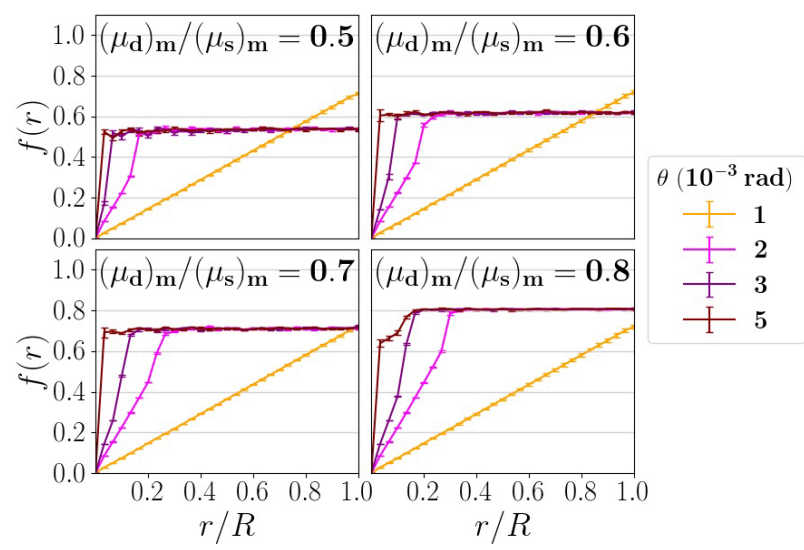

Fig. 6 Stress fields $f(r)$ as a function of radius at different time (rotation angle) snapshots, for various ratios of microscopic friction coefficients. A qualitatively similar behavior has been reported in experimental results [26]. These simulations were performed for $\omega=0.2 \mathrm{rad} / \mathrm{s}$ and $N=120$. 

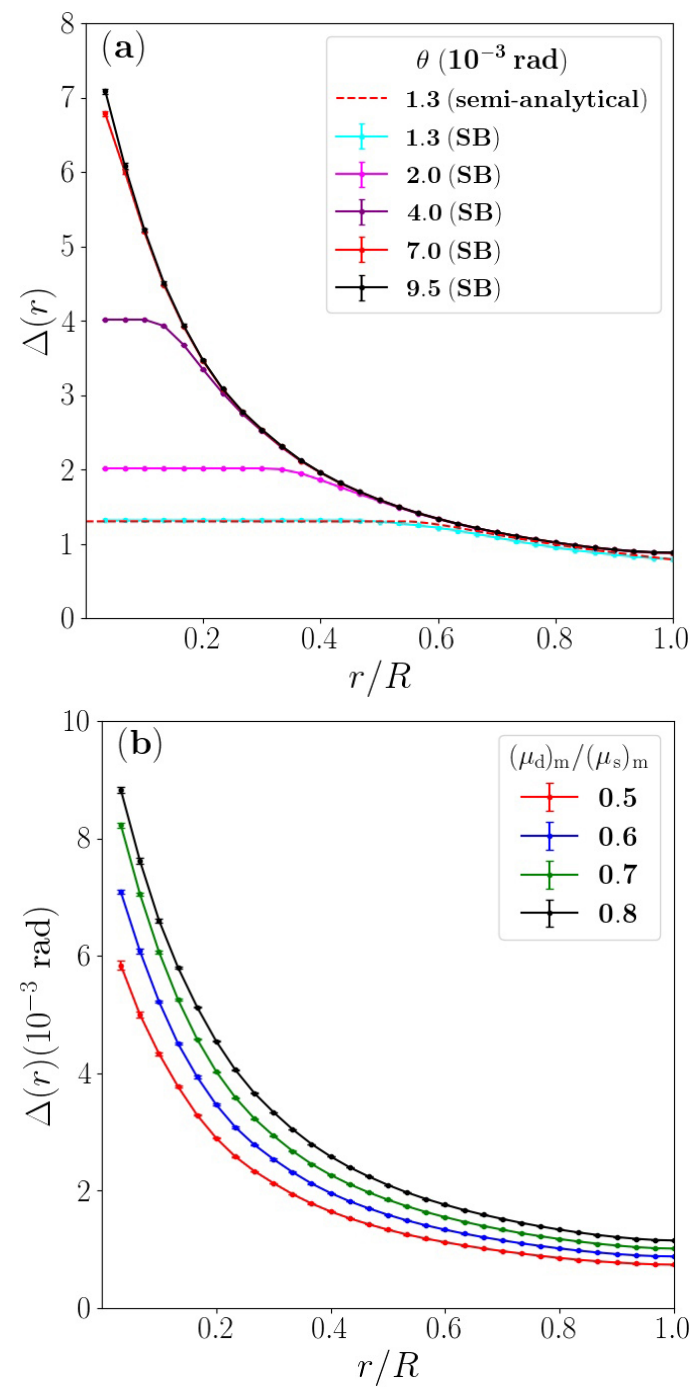

Fig. 7 (a) Angular lag $\Delta(r)$ as a function of the rotation angle for $\left(\mu_{\mathrm{d}}\right)_{\mathrm{m}} /\left(\mu_{\mathrm{s}}\right)_{\mathrm{m}}=0.6$. After the slippage of the external disk region ( $\theta=0.0013 \mathrm{rad})$, the model curve is well fitted by that predicted by semi-analytical calculations. By increasing the rotation angle, the lag increases, until it stabilizes in the dynamic sliding regime, as can be observed from the two largest $\theta$ for which the curves coincide. (b) Angular lag as function of the ratio between static and dynamic friction coefficient. These simulations were performed for $\omega=0.2 \mathrm{rad} / \mathrm{s}$ and $N=120$.

lag behaves as $\Delta(r) \sim 1 / r$ :

$$
\Delta(x)=\frac{\mu_{\mathrm{d}} P L_{z}}{G R} \frac{1}{x}+c
$$

where $x=r / R$ and $c$ is a constant. This is valid in the small thickness regime and soon after the sliding of the external part. Results in Fig. 7(a), for a $\theta$ corresponding to the sliding of the external part, approximate these results very well.
In the dynamic sliding regime, i.e., when all disk points slides, the lag follows the decreasing trend reported in Fig. 7(b). If the ratio of the friction coefficients tends to one, since the initial sliding involves a smaller fraction of the surface, the lag between the center and the circumference is increased. Moreover, the lag for points towards the edge follows with a good approximation the law $\Delta(r) \sim 1 / r$, indicating that the system preserves a memory of the distortion occurred during the first phase of the sliding.

\section{Graded surfaces}

In this section, we show how the axisymmetric transition to sliding is modified by variations of the local mean value of the friction coefficients. In other words, we suppose that the Gaussian distribution adopted to assign the local friction coefficient depends on the surface position and, in particular, its mean value is a function of the disk radial coordinate. This can represent many situations, for example an appropriate grading of the local tribological properties to modify the transition to sliding and the disk distortion during the rotation. This can be useful in applications where torsional friction is present and severe fretting fatigue can occur, due to an axisymmetric stress distribution. In this case, appropriate grading of the local tribological properties can achieve a modification in the evolution of disk sliding during rotation.

In real systems, this can be achieved in various ways. Recent experiments have demonstrated how to manipulate the friction coefficients by means of microtextures, either 3D printed or realized with laser texturing or lithographic techniques, so that it is possible to design and combine different types of microtextures to produce a locally variable friction coefficients along the surface. Local tribological properties can also be modified by means of composite materials, coatings, or a varying polishing of the contact surfaces.

Moreover, a non-uniform local friction can be the result of wear phenomena. In a rotating junction between a harder and a softer material, the presence of asperities on the harder surface can produce circular grooves in the softer one, modifying the local contact properties. Thus, a radial variation of the local friction 
can describe a surface subjected to wear and material losses in axisymmetric configurations. For these reasons, it is useful to study how the behavior of the uniform case is modified by radial gradients of the local friction.

In the following, we omit the subscripts $\mathrm{s}, \mathrm{d}$ for static and dynamic friction coefficients since considerations apply to both separately and, for convenience, we denote with $\mu_{\mathrm{m}}$ the mean value of the Gaussian distribution of Eq. (4) to assign the local friction coefficients. Thus, we suppose that this mean value is a function of the radius, $\mu_{\mathrm{m}}(r)=\bar{\mu}_{\mathrm{m}} \cdot g(r)$, where $\bar{\mu}_{\mathrm{m}}$ is the same mean value corresponding to the uniform case, and $g(r)$ is a radial function whose mean value over the radius is equal to 1 . Standard deviations are fixed to $5 \%$ of the mean value $\mu_{\mathrm{m}}(r)$ and the angular velocity to $\omega=0.2 \mathrm{rad} / \mathrm{s}$.

We consider two types of functions representative of the situations previously described: A linear grading and a sinusoidal grading, as shown in Fig. 8, with a maximum variation $\delta$ with respect to the mean value. In the case of linear grading, $g(r)=1+\delta(2 r / R-1)$, where $\delta$ is the constant slope of the gradient. In the sinusoidal case, $g(r)=1+\delta \cos (f \pi(1-r / R))$, where $f$ is a constant determining the number of oscillations. These functions are defined to reduce the local friction coefficients in the center by a factor $\delta$ and, conversely, to increase them by a factor $\delta$ along the outer circumference, but the overall mean value along the radius is the same of the uniform case.

In the model, this effect can also be achieved by means of a non-uniform pressure distribution, e.g., a linearly increasing load towards the disk edge. However, we prefer to address the grading from the point of view of surface texturing, as in recent experimental approaches [43-45].

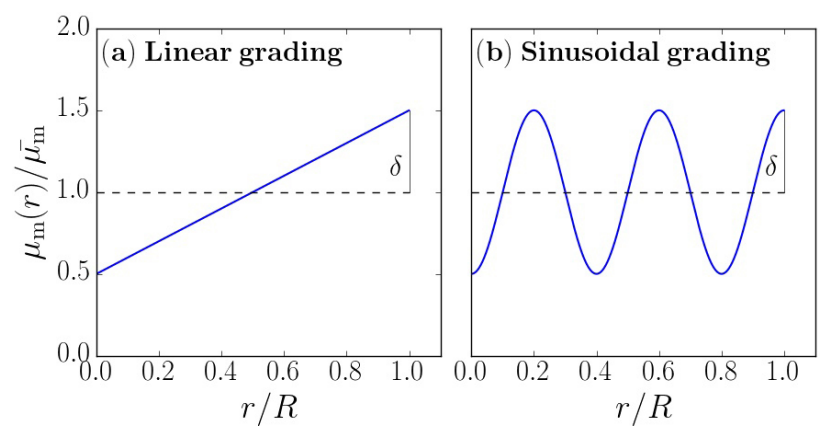

Fig. 8 (a) Linear grading of local friction coefficients with the notation used in the text and (b) sinusoidal grading with $f=5$.

\subsection{Linear gradient}

In the uniform case, the slip of the external part stops before the center at a point where the longitudinal forces are no longer sufficient to exceed the static friction thresholds and trigger a further slip. Thus, if local friction coefficients in the central region of the disk are reduced, we may expect that the sliding proceeds further and spans the whole disk surface at the same time. Moreover, during the rotation, points towards the center slide with a smaller dynamic friction, balancing the smaller torque and, consequently, reducing the angular lag. These effects can be achieved by a linear gradient for both friction coefficients:

$$
\mu_{\mathrm{m}}(r)=\bar{\mu}_{\mathrm{m}}(1+\delta(2 r / R-1))
$$

Thus, friction is increasing from the center to the outer circumference. In Fig. 9(a), we show the critical angles for various $\delta$ values as a function of the radial coordinate for the case $\left(\bar{\mu}_{\mathrm{d}}\right)_{\mathrm{m}} /\left(\bar{\mu}_{\mathrm{s}}\right)_{\mathrm{m}}=0.6$. As expected, the critical radius decreases with $\delta$, so that the area simultaneously slipping at the onset of the sliding increases. For $\delta=0.9$ the whole surface slides abruptly at the same time and, consequently, the difference of angular lag between the center and the edge of the disk is almost zero.

The drawback is that such a sharp transition, triggered by larger friction at the edges, implies a larger static friction peak, as shown in Fig. 9(b) reporting the time evolution of the total torque exerted by the rotating slider. Moreover, in the dynamic rotation regime, the total torque is increased. This is an effect due to the average of the friction coefficients over all the surface. Indeed, with the linear function proposed is not possible to keep simultaneously the same mean value along the radius and on the total area. In the inset of Fig. 9(b), we show all curves divided by the factor $n_{\delta}$ normalizing the total average:

$$
n_{\delta}=\frac{\left(\bar{\mu}_{\mathrm{d}}\right)_{\mathrm{m}}}{\left(\bar{\mu}_{\mathrm{s}}\right)_{\mathrm{m}}}(1+\delta / 3)
$$

The collapse is not perfect as found for the uniform case in Section 3.2 and there is a residual discrepancy increasing for larger $\delta$, which can be ascribed to the larger frictional force contribution of the external part 

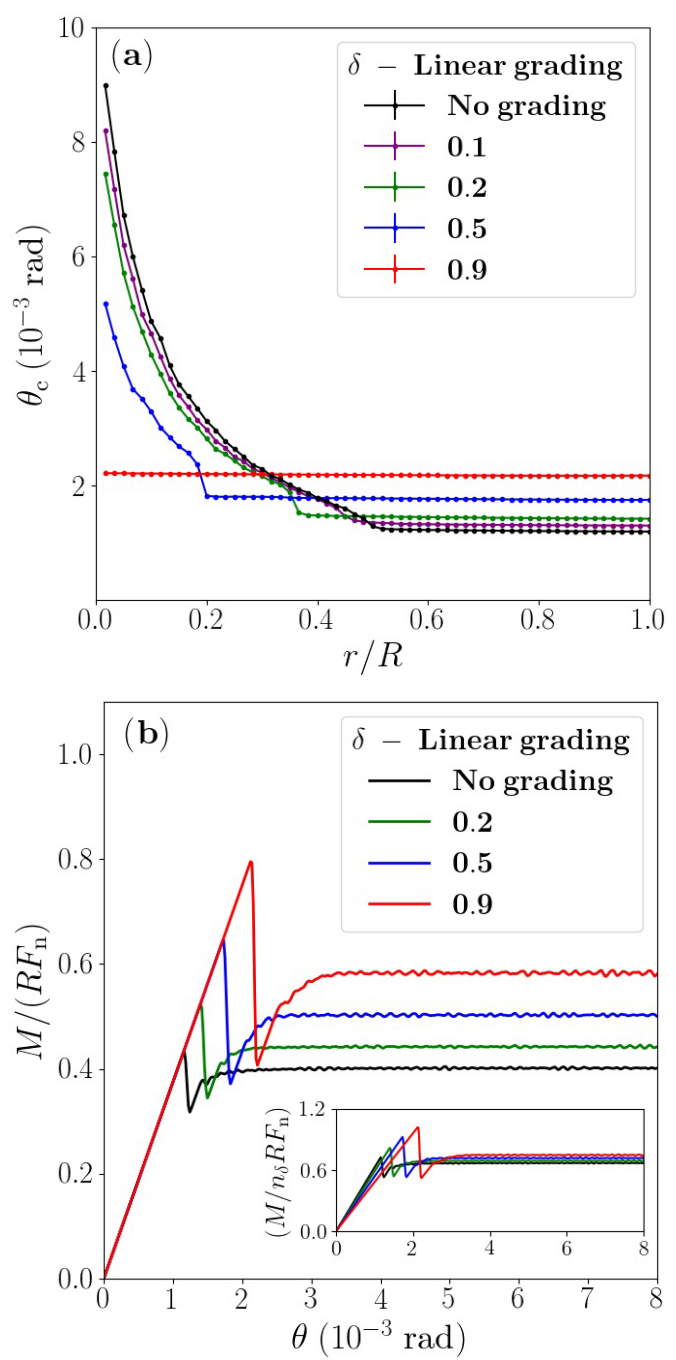

Fig. 9 (a) Critical angle as a function of the radial coordinate of the disk for various grading slopes $\delta$, with a fixed ratio $\left(\bar{\mu}_{\mathrm{d}}\right)_{\mathrm{m}} /\left(\bar{\mu}_{\mathrm{s}}\right)_{\mathrm{m}}=0.6$. (b) Time evolution of the total torque for various $\delta$. In the inset, the same curves are scaled by a factor $n_{\delta}=\left(\bar{\mu}_{\mathrm{d}}\right)_{\mathrm{m}} /\left(\bar{\mu}_{\mathrm{s}}\right)_{\mathrm{m}}(1+\delta / 3)$.

of the disk. Thus, the gradient can be also used to increase the torque in the dynamic rotational sliding.

This behavior can be explained by means of the analytical calculations reported in the Appendix. According to Eq. (6), the lag $\Delta$ in the limit of small thickness is proportional to the inverse of the radius. By replacing $\mu_{\mathrm{d}}$ with Eq. (7), the lag of Eq. (6) becomes

$$
\Delta(x)=\frac{\bar{\mu}_{\mathrm{d}} P L_{z}}{G R} \frac{1-\delta}{x}+2 \delta+\mathrm{c}
$$

Thus, if $\delta$ tends to one, the dependence on $x$ vanishes, so that the lag is approximately constant along the radius.
By varying the ratio between dynamic and static friction coefficients, we find the same qualitative behavior, but all the effects observed in the uniform case are also retained. Thus, by increasing the ratio for a fixed gradient $\delta$, the effect of simultaneous slip is progressively less efficient. In particular, for $\left(\bar{\mu}_{\mathrm{d}}\right)_{\mathrm{m}} /\left(\bar{\mu}_{\mathrm{s}}\right)_{\mathrm{m}}=0.8$, even a $\delta=0.9$, which corresponds to ninety percent of relative reduction at the center, is not sufficient to induce the sliding of the whole surface, although the critical radius is reduced. This is evident from Eq. (9), since for a larger $\bar{\mu}_{\mathrm{d}}$, the reduction of the lag between external and internal part of the disk is less effective.

\subsection{Sinusoidal gradient}

We consider a sinusoidal function $g(r)$, so that both friction coefficients are a function of the radius as follows:

$$
\mu_{\mathrm{m}}(r)=\bar{\mu}_{\mathrm{m}}[1+\delta \cos (f \pi(1-r / R))]
$$

To gain some insight, we focus on the case $f=5$, as in Fig. 8(b). In this way, the disk is characterized by a reduced friction around the center and in two concentric rings. In other regions, friction is increased by the same amount so that the mean value is the same of the uniform case, and $\delta$ is the maximum relative variation with respect to the average.

The critical angle as a function on the radial coordinate is shown in Fig. 10(a). For $\delta<0.2$ the curve is qualitatively similar to the uniform case. Despite the oscillations of the function $g(r)$, the transition to sliding begins from the edge and ends to a critical radius larger than in the uniform case. After this, the rest of the surface slips progressively. However, for a larger $\delta$ this behavior is significantly modified. The sliding begins abruptly from an annular region across the points with smaller friction, so that it is not possible to define a single critical radius. In the subsequent dynamic regime, the sliding propagates to the neighboring areas of these regions and, finally, towards the center. These effects are enhanced for larger $\delta$, so that the angular lag increases.

Thus, for smaller $\delta$ this type of gradient slightly reduces the angular lag but increases the critical radius. For larger $\delta$ values, which is a configuration similar to the case of deep grooves on the disk, it is 

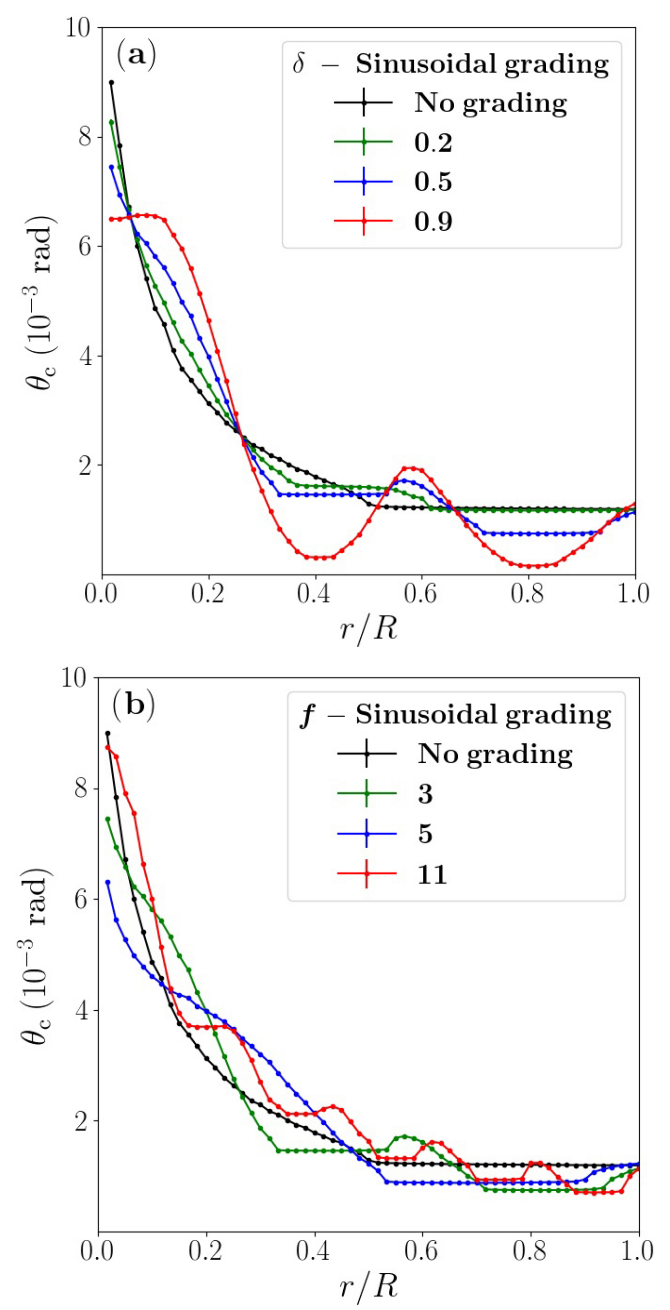

Fig. 10 (a) Critical angle as a function of the radial coordinate of the disk for sinusoidal gradients, with $f=5$ and a fixed ratio $\left(\bar{\mu}_{\mathrm{d}}\right)_{\mathrm{m}} /\left(\bar{\mu}_{\mathrm{s}}\right)_{\mathrm{m}}=0.6$. (b) Critical angle for various $f$ determining the number of oscillations of $g(r)$ for $\delta=0.5$ and $\left(\bar{\mu}_{\mathrm{d}}\right)_{\mathrm{m}} /\left(\bar{\mu}_{\mathrm{s}}\right)_{\mathrm{m}}=0.6$.

totally ineffective to reduce the critical radius and the distortion of the disk during the rotation increases. On the contrary, in time evolution of the total torque, there is smaller peak due to the transition from static to dynamic phase. This is obviously due the transition to sliding nucleating in various separated regions of the disk.

If we change the number of oscillations of $g(r)$, we observe the same phenomenology. In particular, for larger $f$ the transition to sliding has a stepwise shape, as shown in Fig. 10(b), but the angular lag is increased.

\subsection{Friction coefficients}

As reported in Section 2.2, it is useful to define effective friction coefficients to characterize the time evolution of the torque. The effective dynamic friction coefficient, $\left(\mu_{\mathrm{d}}\right)_{M^{\prime}}$ represents the average of the torque after the transition to sliding divided by the radius and the normal force. The effective static coefficient, $\left(\mu_{\mathrm{s}}\right)_{M}$, represents the maximum of the torque in the time range between the beginning and the onset of sliding of the center, so as to take also into account the behavior without a force peak, e.g., Fig. 4(a). In these cases, effective dynamic and static friction coefficients coincide.

In Fig. 11, we compare the variation of these macroscopic quantities for various gradients, obtained for a ratio of the average microscopic friction coefficients $\left(\bar{\mu}_{\mathrm{d}}\right)_{\mathrm{m}} /\left(\bar{\mu}_{\mathrm{s}}\right)_{\mathrm{m}}=0.6$. The values have been normalized by the factor $n_{\delta}$ to take into account the difference between total averages. In the linear case, there is an increasing trend with $\delta$, which is the result of the transition to sliding detailed in the Section 4.1. In the sinusoidal case, the slippage mechanism described in Section 4.2 induces a smooth increase of the macroscopic torque, so that the static friction coefficient tends to the value of the dynamic one for increasing $\delta$. In both cases, the dynamic friction coefficient is increasing with $\delta$, although the relative variation is smaller than $10 \%$, as explained in Section 4.1 .

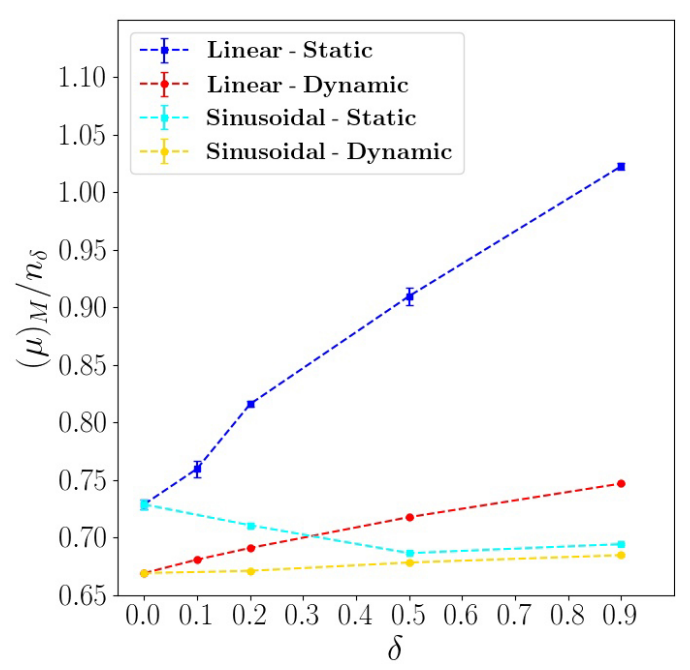

Fig. 11 Comparison between static and dynamic effective friction coefficients as a function of $\delta$ for linear and sinusoidal grading.

\section{Conclusions}

In this paper, we have applied for the first time the framework of the two-dimensional (2D) spring-block 
model to an axisymmetric configuration, describing the case of a thin elastic disk in torsional contact over a rigid substrate. Since this is representative of many experiments and practical applications, we have focused on the description of the transition from static to dynamic friction and on the manipulation of the tribological properties of this setup by an appropriate design of the surface.

We have shown that a simple formulation of the 2D spring-block is able to reproduce qualitative processes observed in experiments at the onset of sliding, allowing to study the time evolution of the surface stress distribution and the resulting macroscopic forces. In particular, due to the axisymmetric configuration, we have highlighted that the transition to sliding is characterized by a sharp slippage of the outer regions of the disk, which stops at a critical radius. Regions inside the critical radius progressively slide during the dynamic rotational regime. Consequently, the disk is deformed, since regions close to the center rotate at larger angular lag those on the edge. This angular lag can lead to non uniform wear and fatigue. We have qualitatively shown how this lag and the critical radius depend on the angular velocity and on the ratio between static and dynamic friction of the materials.

Further, we have analyzed how this behavior can be modified by a grading of the local friction coefficients of the rotating surface. By using a linear grading, the disk distortion caused by the axisymmetric sliding can be reduced and avoided, provided that an appropriate gradient slope is present. We have also considered the case with a sinusoidal grading, mimicking the presence of rings with smaller friction on the disk surface. Their presence is detrimental for the disk deformation and enhances the effect of the regions rotating with larger angular lag. Overall, we have shown how it is possible to use a simple numerical tool such as the spring block model to gain qualitative insights on the phenomenology of the tribological properties of the considered axisymmetric geometry. This can be exploited for the design of modified contact surfaces for enhanced performance in mechanical devices and biomedical implants.

\section{Acknowledgements}

Federico Bosia and Nicola M Pugno (NMP) are supported by the European Commission under the Future Emerging Technologies Open "Boheme" Grant No. 863179. NMP is supported by "Fondazione CARITRO: Laser surface microtexturing for tuning friction". NMP acknowledges the Province of Trento for the financial support provided with the LP 6/99 for the "Meta-F" project. Gianluca Costagliola has received funding from the European union's Horizon 2020 research and innovation programme under the Marie Sklodowska-Curie Grant Agreement No. 754462.

\section{Appendix}

We consider an elastic disk of radius $R$, thickness $L_{z}$, shear modulus $G$. The external part of the disk slips abruptly at the beginning of the transition to sliding, whereas the internal part slides gradually. These regions are divided by a critical radius, namely $R_{\mathrm{c}}$. In order to estimate the critical radius, we write an energy balance. We divide the external part of the disk in thin annuli of width $\mathrm{d} r$ at a distance $r$ from the center, as depicted in Fig. A1.

Before the sliding, the shear elastic energy due to the rotor is stored. We assume that the slippage of the external part and the disk readjustment is instantaneous with respect to the rotation velocity. After the first phase of the transition to sliding, the rotor is rotated by an angle $\theta_{0}$, whereas each annulus of the external part of the disk is rotated from the initial position, but with a smaller angle $\theta(r)$ than $\theta_{0}$ due to the frictional resistance. The difference between the nominal rotation angle $\theta_{0}$ and the angle rotated by the annulus is the angular lag, namely $\Delta(r) \equiv \theta_{0}-\theta(r)$, which is in

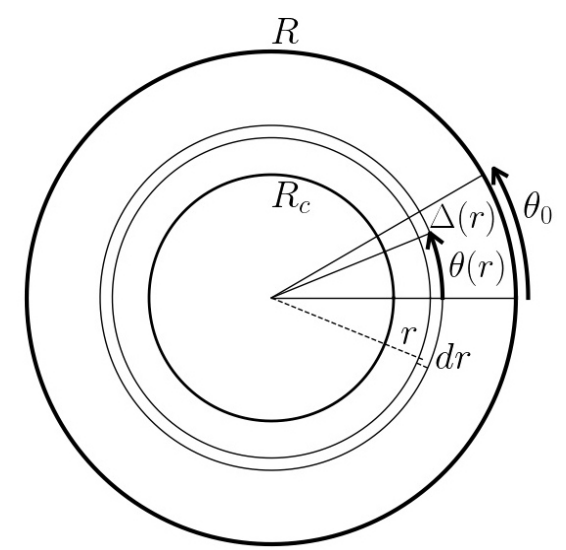

Fig. A1 Schematic of the analytical calculations with the notation used in the text. 
general a non-trivial function of $r$. The internal part is still stuck, so that $\Delta\left(R_{\mathrm{c}}\right)=\theta_{0}$.

Thus, for each annulus of the external part we can write an energy balance before and after the first phase of the transition to sliding. The initial elastic energy due to the rotor has been partially dissipated by friction, is partially still stored in the rotor due to the lag $\Delta(r)$, and is partially stored as shear elastic energy due to the deformation between adjacent annuli.

The total shear elastic energy $U_{\mathrm{e}}^{0}$ before the sliding for an infinitesimal sector $\mathrm{d} S \equiv r \mathrm{~d} r \mathrm{~d} \theta$ of an annulus is $U_{\mathrm{e}}^{0}=\frac{1}{2} G \gamma_{z \theta}^{2} L_{z} \mathrm{~d} S$, where the shear strain $\gamma_{z \theta}$ due to the rotor is $\gamma_{z \theta}=r \theta_{0} / L_{z}$. By integrating over the full angle and between $R_{\mathrm{c}}$ and $R$, we obtain:

$$
\begin{aligned}
& U_{\mathrm{e}}^{0}=\int_{0}^{2 \pi} \int_{R_{\mathrm{c}}}^{R} \frac{1}{2} G L_{z}^{-1} \theta_{0}^{2} r^{3} \mathrm{~d} r \mathrm{~d} \theta \\
& U_{\mathrm{e}}^{0}=\frac{\pi G}{4 L_{z}} \theta_{0}^{2}\left(R^{4}-R_{\mathrm{c}}^{4}\right)
\end{aligned}
$$

After the sliding of the external part, the energy dissipated by friction, $U_{\text {fr }}$, for an annulus can be calculated as the work performed by the friction force: $\theta(r) r \mu_{\mathrm{d}} P \mathrm{~d} S$. By substituting $\theta(r)$ as a function of $\Delta(r)$, the integration leads to:

$$
U_{\mathrm{fr}}=2 \pi \mu_{\mathrm{d}} P \int_{R_{\mathrm{c}}}^{R}\left(\theta_{0}-\Delta(r)\right) r^{2} \mathrm{~d} r
$$

The final elastic energy $U_{\mathrm{e}}^{\mathrm{fin}}$ after the sliding is made of two contributions, the residual elastic energy due to the shear deformation in the vertical plane due to rotor, i.e., $U_{z \theta}$, and the shear energy for an annulus due to its neighbors, i.e., $U_{r \theta}$, accounting for the shear deformation of the disk on the horizontal plane. Therefore, the total elastic energy after the sliding is $U_{\mathrm{e}}^{\mathrm{fin}}=U_{z \theta}+U_{r \theta}$. The first term is similar to the total one, except for the replacement of $\theta_{0}$ with $\Delta(r)$ :

$$
U_{z \theta}=\frac{\pi G}{L_{z}} \int_{R_{c}}^{R} r^{3} \Delta(r)^{2} \mathrm{~d} r
$$

The second term can be written as $U_{r \theta}=\frac{1}{2} G \gamma_{r \theta}^{2} L_{z} \mathrm{~d} S$, where $\gamma_{r \theta}$ is the shear strain of an annulus due to the neighbors. This can be calculated as $\gamma_{r \theta}=r \dot{\Delta}(r)$, where we have indicate with the dot the derivative with respect to $r$. Thus, the integration leads to:

$$
U_{r \theta}=G L_{z} \pi \int_{R_{c}}^{R} r^{3} \dot{\Delta}(r)^{2} \mathrm{~d} r
$$

The total energy balance is: $U_{\mathrm{e}}^{0}=U_{\mathrm{e}}^{\mathrm{fin}}+U_{\mathrm{fr}}$. By substituting the expressions (A3)-(A5), grouping the constants in front of the integral, and defining nondimensional distances $x \equiv r / R$ (the critical radius becomes $\left.x_{\mathrm{c}} \equiv R_{\mathrm{c}} / R\right)$, the energy balance can be written as:

$$
U=\int_{x_{c}}^{1} x^{3} \Delta(x)^{2}+\frac{L_{z}^{2}}{R^{2}} x^{3} \dot{\Delta}(x)^{2}+2 \frac{\mu_{\mathrm{d}} P}{G} \frac{L_{z}}{R} x^{2}\left(\theta_{0}-\Delta(x)\right) \mathrm{d} x
$$

In order to solve this equation, we must find an explicit expression for the function $\Delta(x)$ and the value of $x_{\mathrm{c}}$. After the sliding of the external part, the system readjusts to a new equilibrium position at a minimum of the energy. Thus, for every $x_{c}$, there is a function $\Delta(x)$ minimizing Eq. (A6). Once that the function $\Delta(x)$ is known, the correct $x_{c}$ can be found by imposing that the quantity $U_{\mathrm{e}}^{\mathrm{fin}}+U_{\mathrm{fr}}$ calculated with the integrals in Eqs. (A3)-(A5) corresponds exactly to the initial one $U_{\mathrm{e}}^{0}$. Therefore, we must find the function $\Delta(x)$ so that the following integral assumes the minimum value:

$$
U=\int_{x_{c}}^{1} x^{3} \Delta(x)^{2}+\frac{L_{z}^{2}}{R^{2}} x^{3} \dot{\Delta}(x)^{2}+2 \alpha x^{2}\left(\theta_{0}-\Delta(x)\right) \mathrm{d} x
$$

where we have indicated the parametric constant $\alpha \equiv \mu_{\mathrm{d}} P L_{z} /(G R)$. This problem can be solved by means of the Euler-Lagrange equation for $\Delta(x)$, leading to the differential equation:

$$
\begin{gathered}
\frac{\mathrm{d}}{\mathrm{d} x}\left(\frac{\partial U}{\partial \dot{\Delta}(x)}\right)-\frac{\partial U}{\partial \Delta(x)}=0 \\
\frac{\mathrm{d}}{\mathrm{d} x}\left(2 \frac{L_{z}^{2}}{R^{2}} x^{3} \dot{\Delta}(x)\right)-2 x^{3} \Delta(x)+2 \alpha x^{2}=0
\end{gathered}
$$

By solving this equation with initial conditions $\Delta\left(x_{\mathrm{c}}\right)=\theta_{0}$ and $\dot{\Delta}\left(x_{\mathrm{c}}\right)=0$ to ensure the continuity of the derivative with the internal part where $\Delta(x)=0$, we can find a solution in the range $\left[x_{c}, 1\right]$ for any given $x_{\mathrm{c}}$. Then, the integrals Eqs. (A3)-(A5) can be calculated as a function of $x_{c}$, and the energy balance Eq. (A6) can be imposed to find $x_{\mathrm{c}}$.

The solution of the differential equation, the integration, and the final solution for $x_{c}$ due the energy balance can be solved numerically for the adopted system parameters. We have adopted fourth-order Runge-Kutta method to solve the differential equation. Given the numerical expression of $\Delta(x)$, the integrals Eqs. (A3)(A5) can be calculated with the trapezoidal rule. Finally, 
the algebraic expression originating from Eq. (A6) can be solved with the Newton-Raphson technique to find $x_{c}$ and $\Delta(x)$. This is a semi-analytical approach leading to an approximate numerical solutions, which can be close to the exact one with arbitrary chosen precision. Results of Fig. 4 in the main text have been obtained by dividing the range $\left[x_{c}, 1\right]$ into 10,000 intervals and adopting the mentioned techniques.

However, an analytical approximate solution can be found by observing that the thickness $L_{z}$ of the disk is much smaller than the radius $R$, and that the term with the derivative in Eq. (A7) is smaller than the other due to the factor $\left(L_{z} / R\right)^{2}$. Thus, Eq. (A9) can be solved by neglecting the derivative term, obtaining straightforwardly:

$$
\Delta(x)=\frac{\alpha}{x}+c=\frac{\mu_{\mathrm{d}} P L_{z}}{G R} \frac{1}{x}+c
$$

where $c$ is a constant, which can be fixed by imposing the initial condition $\Delta\left(x_{\mathrm{c}}\right)=\theta_{0}$. A similar parameter combination relevant for the problem, i.e., $\mu_{\mathrm{d}} P / G$, was also found by Lubkin [24].

Equation (A10) is only an approximation and loses the continuous derivative in $x_{c}$, but it allows to perform analytically integrals Eqs. (A3)-(A5). After some calculations of polynomial integrals, a simple expression for the critical radius can be found:

$$
x_{\mathrm{c}}=\frac{\alpha}{2 \theta_{0}}
$$

The rotation angle $\theta_{0}$ can be deduced by considering that the first slipping points are located on the edge of the disk, when the shear force due to the rotor exceeds the static friction force. By equating the two forces and assuming a zero standard deviation for the same static friction thresholds, we find $\theta_{0}=$ $\frac{1}{2} \mu_{\mathrm{s}} P L_{z} /(G R)$. By substituting this into Eq. (A11), we find the direct proportionality $x_{\mathrm{c}}=\frac{\mu_{\mathrm{d}}}{\mu_{\mathrm{s}}}$ discussed in Section 3.2.

This procedure can be also applied in the presence of a gradient of the friction coefficients, provided that the constant $\mu_{\mathrm{d}}$ is replaced with the function $\mu_{\mathrm{d}}(x)$. In particular, for the linear grading of Eq. (7) in Section 4.1, the lag $\Delta(x)$ becomes:

$$
\Delta(x)=\frac{\bar{\mu}_{\mathrm{d}} P L_{z}}{G R} \frac{1-\delta}{x}+2 \delta+c
$$

This demonstrates that, if $\delta$ tends to one, the depen- dence on $x$ vanishes, so that the lag is approximately constant along the radius, as reported in Section 4.1. In this equation, it is also clear that, for a larger $\bar{\mu}_{\mathrm{d}}$, the reduction of the lag between external and internal part of the disk is less effective.

Open Access This article is licensed under a Creative Commons Attribution 4.0 International License, which permits use, sharing, adaptation, distribution and reproduction in any medium or format, as long as you give appropriate credit to the original author(s) and the source, provide a link to the Creative Commons licence, and indicate if changes were made.

The images or other third party material in this article are included in the article's Creative Commons licence, unless indicated otherwise in a credit line to the material. If material is not included in the article's Creative Commons licence and your intended use is not permitted by statutory regulation or exceeds the permitted use, you will need to obtain permission directly from the copyright holder.

To view a copy of this licence, visit http://creativecommons.org/licenses/by/4.0/.

\section{References}

[1] Cai Z B, Zhu M H, Zhou Z R. An experimental study torsional fretting behaviors of LZ50 steel. Tribol Int 43: 361 (2010)

[2] Hager C H, Sanders H, Sharma S. Characterization of mixed and gross slip fretting wear regimes in Ti6Al4V interfaces at room temperature. Wear 257: 167 (2004)

[3] Zhang P, Lu W, Liu X, Zhai W, Zhou M, Zeng W. Torsional fretting and torsional sliding wear behaviors of $\mathrm{CuNiAl}$ against 42CrMo4 under dry condition. Tribol Int 118: 11 (2018)

[4] Fouvry S, Kapsa P, Vincent L. Quantification of fretting damage. Wear 200: 186 (1996)

[5] Zhu M H, Zhou Z R, Kapsa P, Vincent L. An experimental investigation on composite fretting mode. Tribol Int 34: 733 (2001)

[6] Potočnik R, Göncz P, Flasker J, Glodez S. Fatigue life of double row slewing ball bearing with irregular geometry. Procedia Eng 2: 1877 (2010)

[7] Bhaumik S K, Rangaraju R, Venkataswamy M A, Bhaskaran T A, Parameswara M A. Fatigue fracture of crankshaft of an aircraft engine. Eng Fail Anal 9: 255 (2002)

[8] Wang S, Mao Y, Teng B, Pan L. Modeling and simulating of center plate torsion behavior during bogie curve and turnout negotiation. J Mech Sci Technol 28: 2723 (2014) 
[9] Kettler A, Bushelow M, Wilke H J. Influence of the loading frequency on the wear rate of a polyethylene-on-metal lumbar intervertebral disc replacement. Eur Spine J 21: 709 (2012)

[10] Billi F, Sangiorgio S N, Aust S, Ebramzadeh E. Material and surface factors influencing backside fretting wear in total knee replacement tibial components. $J$ Biomech 43 : 1310 (2010)

[11] Xu H, Chen K, Zhang D, Yang X. Torsional friction behavior of the contact interface between the materials of an artificial knee joint replacement, J Biomater Sci Polym 29: 562 (2018)

[12] Bishop N E, Waldow F, Morlock M M. Friction moments of large metal-on-metal hip joint bearings and other modern designs. Med Eng Phys 30: 1057 (2008)

[13] Zhang H, Blunt L, Jiang X, Brown L, Barrans S. The Significance of the micropores at the stem-cement Interface in total hip replacement. J Biomater Sci Polym 22: 845 (2011)

[14] Mathieu V, Vayron R, Barthel E, Dalmas D, Soffer E, Anagnostou F, Haiat G. Mode III cleavage of a coin-shaped titanium implant in bone: Effect of friction and crack propagation. J Mech Behav Biomed Mater 8: 194 (2012)

[15] Cai Z B, Zhang G, Zhu Y, Shen M, Wang L, Zhu M H. Torsional fretting wear of a biomedical Ti6Al7Nb alloy for nitrogen ion implantation in bovine serum. Tribol Int 59: 312 (2013)

[16] Wang S, Wang F, Liao Z, Wang Q, Liu Y, Liu W. Study on torsional fretting wear behavior of a ball-on-socket contact configuration simulating an artificial cervical disk. Mater Sci Eng C 55: 22 (2015)

[17] Bitter T, Khan I, Marriott T, Lovelady E, Verdonschot N, Janssen D. A combined experimental and finite element approach to analyse the fretting mechanism of the headstem taper junction in total hip replacement. Proc Inst Mech Eng H 231: 862 (2017)

[18] Hetenyi M, McDonald P. Contact stresses under combined pressure and twist. Trans ASME J Appl Mech 25: 396 (1958)

[19] Briscoe B J, Chateauminois A. Measurements of frictioninduced surface strains in a steel/polymer contact. Tribol Int 35: 245 (2002)

[20] Chaudhury M K, Chung J Y. Studying friction and shear fracture in thin confined films using a rotational shear apparatus. Langmuir 23: 8061 (2007)

[21] Yu K, Cai Z B, Zhu M H, Qu S, Zhou Z R. Study on torsional fretting behavior of UHMWPE. Appl Surf Sci 255: $616(2008)$

[22] Wang S, Niu C. Torsional Tribological behavior and torsional friction model of polytetrafluoroethylene against 1045 steel. Plos One 11: e0147598 (2016)

[23] Mindlin R. Compliance of elastic bodies in contact. Trans ASME J Appl Mech 16: 259 (1949)

[24] Lubkin J L. The torsion of elastic spheres in contact. Trans ASME J Appl Mech 18: 183 (1951)
[25] Hills D, Sackfield A. The stress field induced by a twisting sphere. Trans ASME J Appl Mech 53: 37222 (1986)

[26] Chateauminois A, Fretigny C, Olanier L. Friction and shear fracture of an adhesive contact under torsion. Phys Rev E 81: 026106 (2010)

[27] Trejo M, Fretigny C, Chateauminois A. Friction of viscoelastic elastomers with rough surfaces under torsional contact conditions. Phys Rev E 88: 052401 (2013)

[28] Dintwa E, Van Zeebroeck M, Tijskens E, Ramon H. Torsion of viscoelastic spheres in contact. Granul Matter 7: 169 (2005)

[29] Hui C Y, Feng X, Jagota A. In situ measurement of the viscoelastic modulus of gels using pure twist-theory. Soft Matter 9: 913 (2013)

[30] Lu W, Zhang P, Liu X, Zhai W, Zhou M, Luo J, Zeng W, Jiang X. Influence of surface topography on torsional fretting wear under flat-on-flat contact. Tribol Int 109: 367 (2017)

[31] Rubinstein S M, Cohen G, Fineberg J. Detachment fronts and the onset of dynamic friction. Nature 430: 1005 (2004)

[32] Rubinstein S M, Cohen G, Fineberg J. Dynamics of Precursors to Frictional Sliding. Phys Rev Lett 98: 226103 (2007)

[33] Braun O M, Barel I, Urbakh M. Dynamics of transition from static to kinetic friction. Phys Rev Lett 103: 194301 (2009)

[34] Capozza R, Rubinstein S M, Barel I, Urbakh M, Fineberg J. Stabilizing stick-slip friction. Phys Rev Lett 107: 024301 (2011)

[35] Trømborg J, Scheibert J, Amundsen D S, Thøgersen K, Malthe-Sørenssen A. Transition from static to kinetic friction: Insights from a 2D model. Phys Rev Lett 107: 074301 (2011)

[36] Capozza R, Pugno N M. Effect of surface grooves on the static friction of an elastic slider. Tribol Lett 58: 35 (2015)

[37] Costagliola G, Bosia F, Pugno N M. Static and dynamic friction of hierarchical surfaces. Phys Rev E 94: 063003 (2016)

[38] Costagliola G, Bosia F, Pugno N M. A 2D model for friction of complex anisotropic surfaces. J Mech Phy Solids 112: 50 (2018)

[39] Murarash B, Itovicha Y, Varenberg M. Tuning elastomer friction by hexagonal surface patterning. Soft Matters 7: 5553 (2011)

[40] Li N, Xu E, Liu Z, Wang X, Liu L. Tuning apparent friction coefficient by controlled patterning bulk metallic glasses surfaces. Sci Rep 6: 39388 (2016)

[41] Maegawa S, Itoigawa F, Nakamura T. Effect of surface grooves on kinetic friction of a rubber slider. Tribol Int 102: 326 (2016)

[42] Wu-Bavouzet F, Cayer-Barrioz J, Le Bot A, Brochard-Wyart F, Buguin A. Effect of surface pattern on the adhesive friction of elastomers. Phys Rev E 82: 031806 (2020)

[43] Gnilitskyi I, Rota A, Gualtieri E, Valeri S, Orazi L. Tribological properties of high-speed uniform femtosecond laser patterning on stainless steel. Lubricants 7: 83 (2019) 
[44] Rosenkranz A, Grützmacher P G, Gachot C, Costa H L. Surface texturing in machine elements-A critical discussion for rolling and sliding contacts. Adv Eng Mater 21: 1900194 (2019)

[45] Berardo A, Costagliola G, Ghio S, Boscardin M, Bosia F, Pugno N M. An experimental-numerical study of the adhesive static and dynamic friction of micropatterned soft polymer surfaces. Materials \& Design 181: 107930 (2019)

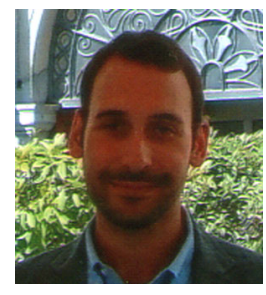

Gianluca COSTAGLIOLA. He received his master degree in physics at the University of Pisa, Italy, in 2011 and a Ph.D. in physics in 2015 at the University of Torino. He worked as postdoc researcher at the University of Torino from 2015 to 2019, and

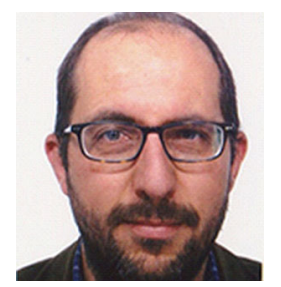

Federico BOSIA. He graduated in physics at the University of Torino in 1996 and obtained a Ph.D. in mechanical engineering in 2002 at EPFL (Switzerland). He has worked as research assistant and postdoc at the University of Roma "La Sapienza" (Italy), the Leibniz Institute for Solid State and Materials Research Dresden (Germany), and the Politecnico di Torino (Italy). He worked at the University of Torino from 2007 to 2019 and is assistant professor at Politecnico di Torino since 2020. He has published about 110

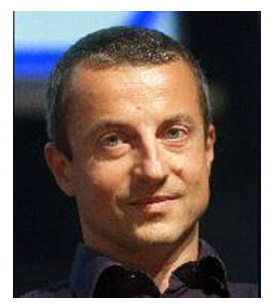

Nicola M PUGNO. He was born in 1972. He obtained master degrees in engineering and physics (both cum laude) and Ph.D. degrees in engineering and biology. He is a professor of solids and structural mechanics at the University of Trento since 2012 and a director of the Laboratory for Bioinspired, Bionic, Nano, Meta Materials \& Mechanics there (previously founded at Politecnico di Torino as Laboratory of Bio-Inspired Nanomechanics Giuseppe Maria Pugno), and a professor of material science at the Queen Mary University of London since 2013 (part-time). He has published about 500 papers in international journals. He is a plenary and invited speaker in
[46] Costagliola G, Bosia F, Pugno N M. Tuning friction with composite hierarchical surfaces. Tribol Int 115: 261 (2017)

[47] Guarino R, Costagliola G, Bosia F, Pugno N M. Evidence of friction reduction in laterally graded materials. Beilstein J Nanotechnol 9: 2443 (2018)

[48] Absi E, Prager W. Comparison of equivalence and finite element methods. Comp Methods in Appl Mech Eng 6: 59 (1975)

he is currently employed at the École Polytechnique Fédérale de Lausanne (EPFL), Switzerland, as postdoc holding a Eurotech fellowship, under the Marie Sklodowska-Curie grant programme. His research is focused on numerical simulations applied to material science, particularly in the field of tribology and fracture mechanics.

papers in leading international journals. $\mathrm{He}$ is currently responsible for PoliTo for the Future Emerging Technologies-Open project BOHEME. He was previously involved as additional participant in an European Research Council Starting grant on bioinspired materials and as third party in a FET Proactive grant project on the design of devices for regeneration of spinal cord injuries. He has experience in both experimental mechanics of composite materials and numerical multiscale modelling of fracture and elastic wave propagation.

several international conferences (including Falling Walls, World Economic Forum and at the European Parliament, invited by the European Research Council-ERC, opening plenary lecturer at International Congress of Theoretical and Applied Mechanics $2020+1)$. He is an academic editor of PLOS ONE and of Scientific Reports among several others and first editor-in-chief of Frontiers in Materials and its section on Mechanics. He is a winner of 4 ERC (1 StG and 3 PoC) and other EU grants, such as 1 FET Proactive, 1 FET Open (as coordinator), Graphene Flagship (rampup, core 1, 2, and 3 phases), and national grants. $\mathrm{He}$ is also a winner of the Griffith Medal and Prize 2017. See details at http://www.ing.unitn.it/ pugno/. 